\title{
Vorsicht
}

\section{Digitale Rechnungen rufen Fiskus auf den Plan}

\author{
Elektronische Rechnungen werden immer mehr zur Selbstverständlichkeit. \\ Dabei wird oft übersehen, dass auch der Rechnungsempfänger strenge Re- \\ geln beachten muss, sonst droht Ärger mit dem Fiskus.
}

W ährend Rechnungsaussteller vor Start des „E-Invoicing“ - der digitalen Rechnungsstellung eingehende Vorbereitungen treffen müssen, flattern digitale Abrechnungen Empfängern zum Teil unvermittelt ins Haus. Empfänger elektronischer Rechnungen sollten die steuerlichen Pflichten jedoch keinesfalls unterschätzen. Der Empfang elektronischer Abrechnungsdokumente birgt einige steuerliche Fallstricke, die zu Steuernachzahlungen oder Bußgeldern führen können.

\section{Archivierung auf Papier reicht nicht}

Grundsätzlich müssen digitale Rechnungen die gleichen formalen Kriterien erfüllen wie Papierrechnungen. Leicht werden bei der Belegprüfung am Bildschirm Fehler übersehen. Es ist daher sinnvoll, allen Praxismitarbeitern, die die Rechnungen prüfen, Checklisten an die Hand zu geben, mit denen sie die formale und inhaltliche Richtigkeit lückenlos prüfen können. Darüber hinaus müssen Rechnungsempfänger die Echtheit der Herkunft und die Unversehrtheit des Dokuments sicherstellen. Dies können eine elektronische Signatur oder die Übermittlung per Electronic Data Interchange(EDI)-Verfahren automatisch gewährleisten.

Auch die Archivierung digitaler Rechnungen erfordert erhöhte Aufmerksamkeit. Der Ausdruck eines digitalen Dokuments auf Papier und die anschließende Belegablage reichen aus Sicht der Finanzverwaltung für Archivierungszwecke nicht aus. Eine elektronische Rechnung muss grundsätzlich in dem Datenformat aufbewahrt werden und jederzeit lesbar sein, in dem sie empfangen wurde. Betriebe - also auch Arztpraxen - müssen somit die Softwareprogramme zur Anzeige und Auswertung der Dateien während der gesetzlichen Aufbewahrungspflicht von zehn Jahren vorhalten. Andernfalls kann es spätestens bei einer Betriebsprüfung zu bösen Überraschungen kommen.

\section{Vorsicht mit Buchungsvermerken}

Viele Rechnungssteller versenden Abrechnungen per E-Mail. Bisweilen enthält auch diese steuerrelevante Daten wie etwa einen Hinweis auf Skonto. Dann müssen Rechnungsempfänger nicht nur die digitale Rechnung, sondern auch die E-Mail mit allen Anhängen und Verknüpfungen aufbewahren. Verstöße gegen die Aufbewahrungsvorschriften gelten als Ordnungswidrigkeit. Bei nachlässiger Handhabung ist der Vorsteuerabzug gefährdet. Zudem können die Finanzbehörden Geldbußen von bis zu $5.000 €$ verhängen.Auch für die Bearbeitung eingehender digitaler Rechnungen machen die Finanzbehörden strenge Vorgaben. Das Anbringen von Informationen wie Buchungsvermerken, Indexierungen oder Barcodes darf keinen Einfluss auf die Lesbarkeit des Originalzustands haben.

Die Betriebe müssen alle elektronischen Bearbeitungsvorgänge protokollieren und mit dem digitalen Dokument abspeichern. Nur so ist die Nachvollziehbarkeit und Prüfbarkeit des Originals und seiner Ergänzungen gewährleistet.

\section{Versteckt in den AGBs}

Praxen müssen elektronische Rechnungen aber nicht zwangsläufig dulden. Der Rechnungsaussteller muss vorab das Einverständnis des Empfängers einholen. Doch Vorsicht: Eine Zustimmung kann auch durch die Anerkennung der Allgemeinen Geschäftsbedingungen erfolgen, die den Versand digitaler Rechnungen einschließt.

Torsten Lambertz, Wirtschaftsprüfer und Steuerberater der Kanzlei WWS Wirtz, Walter, Schmitz, in Mönchengladbach (www.wws-gruppe.de). Seine Tätigkeitsschwerpunkte liegen in steuerlichen und betriebswirtschaftlichen Beratungen.

\section{Hier steht eine Anzeige.}

